\title{
Real-Time Observations of Structural Ordering in Graphene Oxide During Thermal Reduction in Vacuum
}

\author{
E.C. Mattson,* S.M. Cui,* M.A. Schofield,* G.H. Lu,** H. Pu,** M.T. Weinert,* J.H. Chen,** C.J. \\ Hirschmugl,* and M. Gajdardziska-Josifovska* \\ *Dept. of Physics, University of Wisconsin-Milwaukee, Milwaukee, WI 53211 \\ **Dept. of Mechanical Engineering, University of Wisconsin-Milwaukee, Milwaukee, WI 53211
}

Since its first isolation in 2004 [1], graphene has intrigued scientists spanning many disciplines. While graphene is a promising candidate for electronic technologies with unprecedented novelty, its large scale production has met challenges. One promising route for large scale synthesis of Graphene-based materials is through the reduction of graphene oxide (GO). Tuning of reduction treatments, however, has been complicated due to the unusual nature of this material. GO is partially disordered, nonstoichiometric, and has varying characteristics based on the synthesis method. The exact structures of $\mathrm{GO}$ and reduced GO are at present not well understood.

In this work, thermal reduction of GO multilayers, as described previously [2], has been examined by in situ electron diffraction studies performed in a H9000 NAR Transmission Electron Microscope (TEM) with an accelerating voltage of $300 \mathrm{kV}$. By recording Selected Area Diffraction (SAD) movies as function of temperature, the structural dynamics of the reduction and resulting material has been elucidated. Further characterization of the materials by synchrotron-based Infrared (IR) Microspectroscopy has been performed on the same samples used for TEM. Utilization of normal and grazing incidence geometries provides insight into geometrical bonding relationships, detecting dynamic dipole moments parallel and perpendicular to the GO basal plane, respectively.

Analysis of unreduced GO normal and grazing incidence IR absorbance spectra (Fig. 1) indicates the presence of oxygen species including hydroxyl $\left(3200 \mathrm{~cm}^{-1}\right.$, normal, $1125 \mathrm{~cm}^{-1}$, grazing), intercalated water (1610 $\mathrm{cm}^{-1}$, grazing), carbonyl $\left(1730 \mathrm{~cm}^{-1}\right.$, normal and grazing), epoxide/ether $\left(1225 \mathrm{~cm}^{-1}\right.$, normal), and carboxyl (1425 $\mathrm{cm}^{-1}$, normal) functional groups, in the bonding scheme of Fig. 2. An SAD pattern of unreduced GO is shown in Fig. 3a. Previous analysis [2] of such patterns indicated that the samples consist of randomly oriented, oxidized carbon monolayers, lacking any oxygen superlattice ordering. Structural evolution during reduction, best illustrated in real-time SAD movies to be shown during presentation, is also demonstrated by still-frame SAD patterns of films quenched from $550^{\circ} \mathrm{C}$ and $650^{\circ} \mathrm{C}$ (Figs. 3b-c). The patterns show that the scattered intensity from the amorphous species is predominantly transferred to two new crystalline rings, and the remaining diffusely scattered intensity moves closer to the reciprocal space origin. This suggests that oxygen species bonded at disordered sites are transformed in favor of oxygen species bonded at ordered sites. The spacings of the new crystalline rings correspond to real-space distances of 2.54 and $1.46 \AA$, which are significant distances in the graphene lattice but not present in graphene diffraction patterns.

The formation of the new diffraction rings in Fig. 3 is correlated to the removal of hydroxyl groups in the IR spectra in Fig 4 from reduced samples quenched from different temperatures. The only IR bands remaining in the absorbance spectra from samples annealed in excess of $650^{\circ} \mathrm{C}$ are assigned to epoxides/ethers $\left(1200 \mathrm{~cm}^{-1}\right)$ and C-C stretching $\left(1600 \mathrm{~cm}^{-1}\right)$, which has developed an asymmetric Fano lineshape [3], attributed to strong coupling of this optical phonon mode to a continuum of direct electronic transitions in the same energy range. Furthermore, the background IR absorbance due to electronic absorption, as well as the linewidth of the Fano resonance, evolve with temperature. From 
these data, the most likely structure contains ether/epoxide groups bonded to produce a hexagonal symmetry within the carbon monolayers. This ordering develops as a response to destabilization induced by the desorption of hydroxyls. The observation of a temperature-dependant Fano resonance and available electronic states between $0.18-0.21 \mathrm{eV}$ indicates that the resulting material has attractive and tunable electronic properties.

\section{References}

[1] Novoselov, K.S. et. al. Science 306 (2004) 666

[2] Mattson, E.C. et. al. Microscopy and Microanalysis 16 (2010) 1706 (and references therein)

[3] U. Fano Phys. Rev. 124 (1961) 1866

[4] This work was supported by the NSF under grant CMMI-0856753. The IR work was performed at the Synchrotron Radiation Center in Stoughton, WI, supported by the NSF under grant DMR-0537588

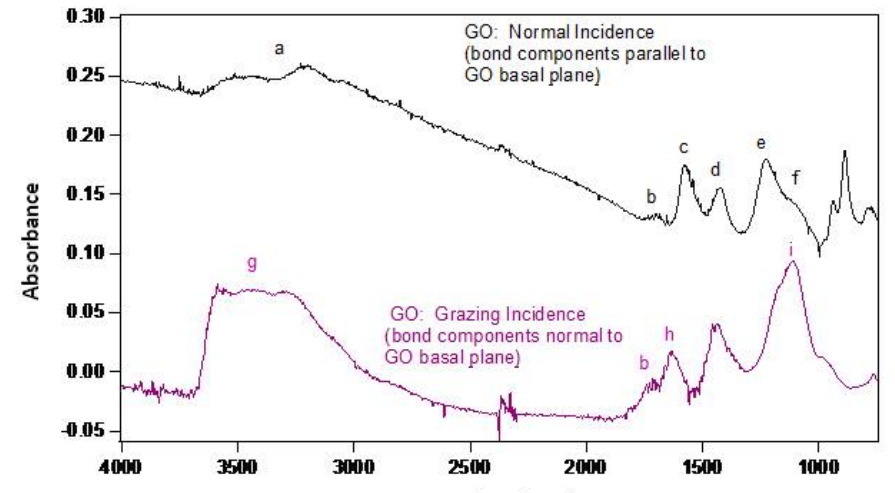

Wavenumbers $\left(\mathrm{cm}^{-1}\right)$
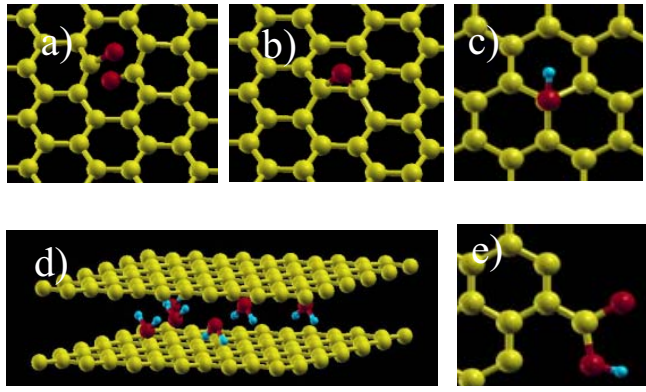

Figure 2: Bonding of oxygen functional groups to carbon lattice in $\mathrm{GO}:(1,2)$ epoxides (a), carbonyls (b), carboxyls (c), intercalated water (d), and hydroxyls (e)
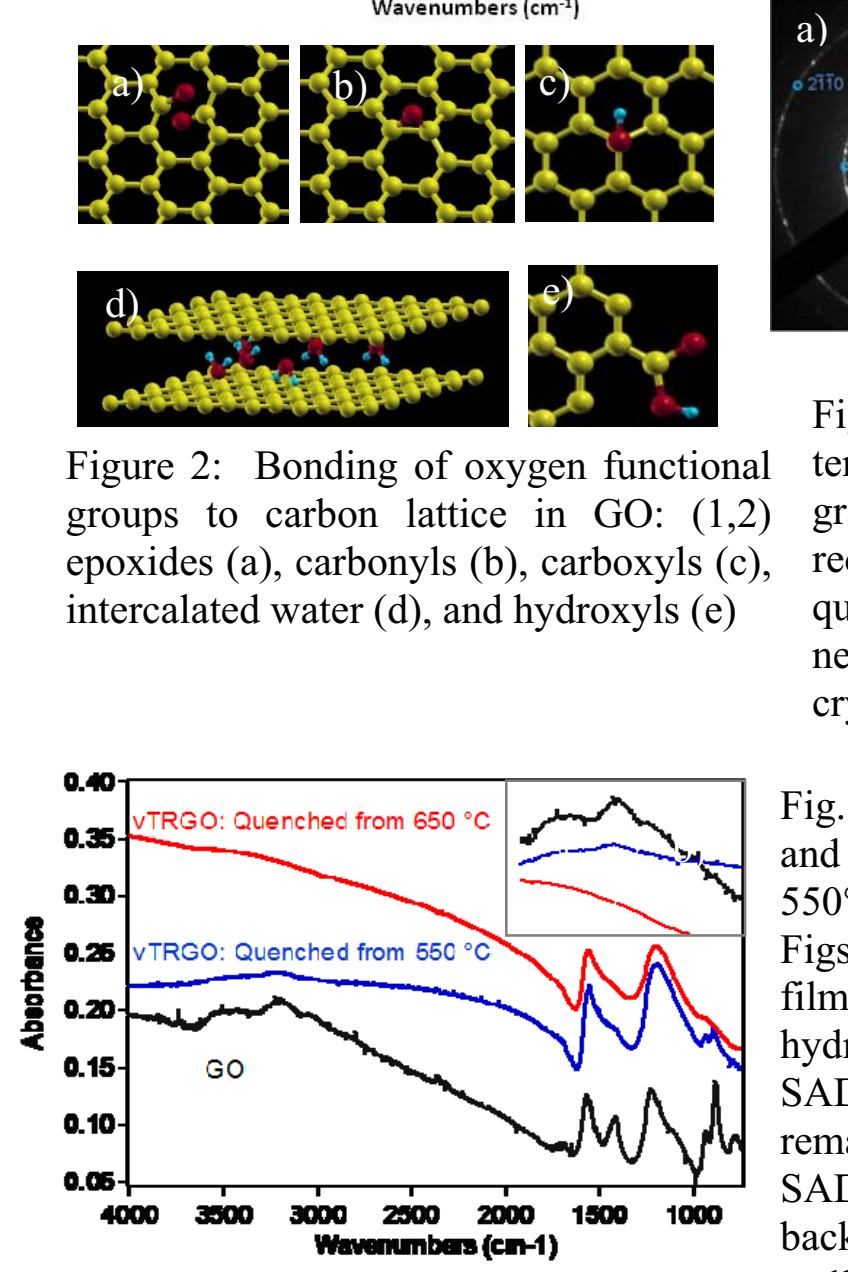

Figure 1: a) IR absorbance of GO measured at normal incidence (top) and grazing incidence (bottom). Peaks are assigned to hydroxyl $\mathrm{O}-\mathrm{H}$ stretching (a), $\mathrm{C}=\mathrm{O}$ stretching (b), $\mathrm{C}=\mathrm{C}$ stretching (c), Carboxyl C-O stretching (d), epoxide/ether $\mathrm{C}-\mathrm{O}-\mathrm{C}$ asymmetric stretch (e), alkoxy $\mathrm{C}-\mathrm{O}$ stretching (f), $\mathrm{H}_{2} \mathrm{O} \mathrm{O}-\mathrm{H}$ stretching $(\mathrm{g}), \mathrm{H}_{2} \mathrm{O} \mathrm{O}-\mathrm{H}$ bending (h), and hydroxyl C-O stretching (i).
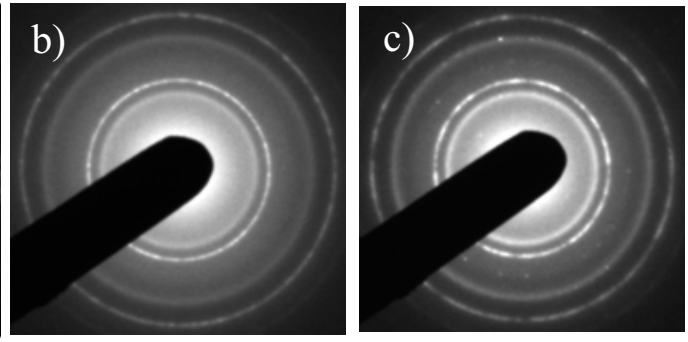

Figure 3: Evolution of GO SAD pattern with temperature: Unreduced GO with indexed monolayer graphene SAD pattern (a) develops two new rings in reduced $\mathrm{GO}$ quenched from $550^{\circ} \mathrm{C}(\mathrm{b})$. Annealing and quenching from $650 \mathrm{C}$ shows increased intensity of the new rings and evidences the enhancement of the crystallinity of the new phase (c).

Fig. 4: a) Normal incidence IR absorption spectra of GO and vacuum thermally reduced (vTR) GO quenched from $550^{\circ} \mathrm{C}$ and $650^{\circ} \mathrm{C}$, corresponding to the $\mathrm{SAD}$ patterns of Figs. $3 \mathrm{~b}$ and $\mathrm{c}$. Inset shows $\mathrm{O}-\mathrm{H}$ stretching region. As the film is annealed to $550^{\circ} \mathrm{C}$, only ethers/epoxides and some hydroxyls remain, in conjunction with formation of new $\mathrm{SAD}$ rings. As the temperature is increased to $650^{\circ} \mathrm{C}$, the remaining hydroxyls are driven away (inset), and the new SAD rings are enhanced. Evolution of the electronic background absorption and Fano lineshape are observed as well. 